\title{
Game Theoretic Approaches to Weight Assignments in Data Envelopment Analysis Problems
}

\author{
Satoru Sekine, Jing Fu, and Shigeo Muto \\ Department of Social Engineering, Tokyo Institute of Technology, 2-12-1 Ookayama, Meguro-ku, Tokyo 152-8550, Japan \\ Correspondence should be addressed to Jing Fu; fu.j.aa@m.titech.ac.jp
}

Received 30 December 2013; Accepted 6 March 2014; Published 9 April 2014

Academic Editor: Changzhi Wu

Copyright (C) 2014 Satoru Sekine et al. This is an open access article distributed under the Creative Commons Attribution License, which permits unrestricted use, distribution, and reproduction in any medium, provided the original work is properly cited.

\begin{abstract}
This paper deals with the problem of fairly allocating a certain amount of divisible goods or burdens among individuals or organizations in the multicriteria environment. It is analyzed within the framework of data envelopment analysis (DEA). We improve the game proposed by Nakabayashi and Tone (2006) and develop an alternative scheme by reassigning the total weight or power for the coalition members. The solutions and equilibria of the new DEA game proposed in this paper are also studied.
\end{abstract}

\section{Introduction}

Recently game theoretic approaches to DEA problems have been often observed. These include, in part, Nakabayashi and Tone [1], Liang et al. [2], Wu et al. [3], Wu et al. [4], and Zhu [5]. Among them, Nakabayashi and Tone [1] studied the problem of allocating a fixed amount of reward to players who are evaluated by multiple criteria. They proposed a new scheme for allocating the reward to the players based on cooperative game theory and DEA. Later, Wu et al. [3] and Wu et al. [4] applied the game model by Nakabayashi and Tone [1] to evaluate the cross efficiency of players (decisionmaking units (DMU) in DEA terms) by using solutions in cooperative games, the nucleolus, and the Shapley value.

The game proposed by Nakabayashi and Tone [1] was, however, subadditive. Namely, players lose their power when they cooperate. The reason is clear. Before forming a coalition, each player has a weight of one and puts it on his/her most preferable criterion. To increase their bargaining power, some of the players choose to form a coalition, but, in Nakabayashi and Tone's [1] assumption, the coalition is only given a weight of one in total though each of the members' precoalition weight was one, which causes the subadditivity. To make the game superadditive, they took the dual of the game, called the DEA min game, and studied solutions such as the core, the Shapley value, and the nucleolus. In the DEA min game, each player and each coalition pick up the weight that minimizes their evaluation. No reasonable justification was given in their paper for picking up the minimizing weight under the assumption that players are egoistic and want to maximize their own evaluation. The purpose of this paper is to propose an alternative, and more natural, cooperative game scheme that fits for the problem. We will start with a strategic form game describing the problem posed by Nakabayashi and Tone [1] and then construct a cooperative game from the strategic form game based on the procedures by von Neumann and Morgenstern [6].

The rest of the paper will be organized as follows. In Sections 2 and 3, we review the DEA problem and the DEA game proposed by Nakabayashi and Tone [1]. After giving a definition of the strategic form game in Section 4, we will present a cooperative TU game representation in Section 5 and then study its properties and solutions in Sections 6 and 7. The Nash equilibrium, strong Nash equilibrium, and coalition-proof Nash equilibrium for the strategic form game are analyzed in Section 8. Section 9 will close the paper with remarks.

\section{The Model}

Let $E(>0)$ denote the fixed amount of benefit to be allocated to players $1, \ldots, n$. Players' contributions are evaluated by multiple criteria and summarized as the score matrix $C=\left(c_{i j}\right)_{i=1, \ldots, m, j=1, \ldots, n}$, where $c_{i j}$ is player $j$ 's contribution 
measured by criterion $i$, called the evaluation index. The problem is to find a weight vector on the criteria determined endogenously by players themselves. Following the DEA analysis, each player $k$ chooses a nonnegative weight vector $w^{k}=\left(w_{1}^{k}, \ldots, w_{m}^{k}\right)$ such that $\sum_{i=1}^{m} w_{i}^{k}=1, w_{i}^{k} \geq 0 \forall i=$ $1, \ldots, m$, where $w_{i}^{k}$ is the weight given to criterion $i$ by player $k$. Then the contribution of player $k$ relative to the total contribution of all players measured by the weight vector $w^{k}$ is given by

$$
\frac{\sum_{i=1}^{m} w_{i}^{k} c_{i k}}{\sum_{i=1}^{m} w_{i}^{k}\left(\sum_{j=1}^{n} c_{i j}\right)}
$$

Player $k$ chooses the weight vector that maximizes this ratio. The weight vector is found by solving the following fractional program:

$$
\begin{aligned}
\max _{w^{k}} & \frac{\sum_{i=1}^{m} w_{i}^{k} c_{i k}}{\sum_{i=1}^{m} w_{i}^{k}\left(\sum_{j=1}^{n} c_{i j}\right)} \\
\text { s.t. } & \sum_{i=1}^{m} w_{i}^{k}=1, \quad w_{i}^{k} \geq 0 \forall i=1, \ldots, m .
\end{aligned}
$$

Each of the other players similarly maximizes the ratio produced by his/her own weight vector.

This maximization problem can be reformulated as the following much simpler form. First for each row $\left(c_{i 1}, \ldots, c_{i n}\right)$, $i=1, \ldots, m$, divide each element by the row-sum $\sum_{j=1}^{n} c_{i j}$. By Charnes-Cooper transformation [7], the maximization problem above is not affected by this operation. Let

$$
c_{i j}^{\prime}=\frac{c_{i j}}{\sum_{j=1}^{n} c_{i j}}, \quad i=1, \ldots, m .
$$

The matrix $C^{\prime}=\left(c_{i j}^{\prime}\right)_{i=1, \ldots, m, j=1, \ldots, n}$ is called the normalized score matrix and $\sum_{j=1}^{n} c_{i j}^{\prime}=1$ is satisfied. Then

$$
\begin{aligned}
& \frac{\sum_{i=1}^{m} w_{i}^{k} c_{i k}}{\sum_{i=1}^{m} w_{i}^{k}\left(\sum_{j=1}^{n} c_{i j}\right)} \\
& =\frac{\sum_{i=1}^{m} w_{i}^{k} c_{i k} / \sum_{j=1}^{n} c_{i j}}{\sum_{i=1}^{m} w_{i}^{k}\left(\sum_{j=1}^{n} c_{i j}\right) / \sum_{j=1}^{n} c_{i j}} \\
& =\frac{\sum_{i=1}^{m} w_{i}^{k} c_{i k}^{\prime}}{\sum_{i=1}^{m} w_{i}^{k}} .
\end{aligned}
$$

Due to $\sum_{i}^{m} w_{i}^{k}=1$, the fractional maximization program above can be expressed as the following linear maximization program:

$$
\begin{array}{ll}
\max _{w^{k}} & \sum_{i=1}^{m} w_{i}^{k} c_{i k}^{\prime} \\
\text { s.t. } & \sum_{i}^{m} w_{i}^{k}=1, \quad w_{i}^{k} \geq 0 \forall i=1, \ldots, m .
\end{array}
$$

Let $c(k)$ be the maximal value of the program. Apparently the maximal value is attained by letting $w_{i(k)}^{k}=1$ for the criterion $i(k)$ such that $c_{i(k) k}^{\prime}=\max _{i=1, \ldots, m} c_{i k}^{\prime}$ and letting $w_{i}^{k}=$ 0 for all other criteria $i \neq i(k)$. Thus $c(k)$ is the highest relative contribution of player $k$. Namely,

$$
c(k)=\max _{i=1, \ldots, m} c_{i k}^{\prime} .
$$

Nakabayashi and Tone [1] showed that if each player $k$ claims the portion $c(k)$ of $E$, the sum of the claims generally exceeds the total benefit $E$. Then the following problem arises: how to allocate $E$ reasonably to players? To find a fair allocation of $E$, they proposed to apply cooperative game theory. Let us review their cooperative game model that they call a DEA game.

In the following sections, we assume that the score matrix is given in the normalized form. That is, $C=$ $\left(c_{i j}\right)_{i=1, \ldots, m, j=1, \ldots, n}$, where $\sum_{j=1}^{n} c_{i j}=1 \forall i=1, \ldots, m ; c_{i j} \geq$ $0 \forall i=1, \ldots, m, \forall j=1, \ldots, n$. Then the fractional maximization program can be rerepresented by

$$
\begin{array}{ll}
\max _{w^{k}} & \sum_{i=1}^{m} w_{i}^{k} c_{i k} \\
\text { s.t. } & \sum_{i}^{m} w_{i}^{k}=1, \quad w_{i}^{k} \geq 0 \forall i=1, \ldots, m .
\end{array}
$$

\section{The DEA Game by Nakabayashi and Tone}

Nakabayashi and Tone [1] construct a characteristic function form game $(N, c)$ in the following manner. $N=\{1, \ldots, n\}$ is the set of players and $c$ is the characteristic function that gives each coalition a value it obtains. For each single player coalition $\{k\}, c(\{k\})$ is given by $c(k) E$. Similarly for each coalition $S \subseteq N, c(S)$ is given by the maximum value of the linear maximization program:

$$
\begin{array}{ll}
\max & \sum_{i=1}^{m}\left(w_{i}^{S} \sum_{j \in S} c_{i j}\right) E \\
\text { s.t. } & \sum_{i=1}^{m} w_{i}^{S}=1, \quad w_{i}^{S} \geq 0 \forall i=1, \ldots, m,
\end{array}
$$

where $w^{S}=\left(w_{1}^{S}, \ldots, w_{m}^{S}\right)$ is the weight vector chosen by $S$. Here they assume that players' evaluation is transferable and take a total of players' evaluation in coalition $S$. Hereafter we call this characteristic function form game NT (Nakabayashi and Tone) DEA game.

Nakabayashi and Tone [1] show a counterintuitive fact that the NT DEA game is subadditive. Namely, players lose their value by forming a coalition. The reason is quite simple. Consider the score matrix in Table 1.

It is easily seen that the optimal weight vector for each player is $(1,0,0)$ for $1,(0,1,0)$ for 2 , and $(0,0,1)$ for 3 , and thus $c(\{1\})=0.6, c(\{2\})=0.7$, and $c(\{3\})=0.8$. Suppose that players 1 and 2 form a coalition and aim at maximizing their joint evaluation for higher bargaining power. Then we have the score matrix in Table 2. 
TABLE 1: Score matrix for single player coalition case.

\begin{tabular}{lcccc}
\hline & Player 1 & Player 2 & Player 3 & Row-sum \\
\hline Criterion 1 & $6 / 10$ & $2 / 10$ & $2 / 10$ & 1 \\
Criterion 2 & $2 / 10$ & $7 / 10$ & $1 / 10$ & 1 \\
Criterion 3 & $1 / 10$ & $1 / 10$ & $8 / 10$ & 1 \\
\hline
\end{tabular}

TABLE 2: Score matrix for the coalition $\{1,2\}$ case.

\begin{tabular}{lccc}
\hline & Coalition $\{1,2\}$ & Player 3 & Row-sum \\
\hline Criterion 1 & $8 / 10$ & $2 / 10$ & 1 \\
Criterion 2 & $9 / 10$ & $1 / 10$ & 1 \\
Criterion 3 & $2 / 10$ & $8 / 10$ & 1 \\
\hline
\end{tabular}

Coalition $\{1,2\}$ 's optimal weight vector is $(0,1,0)$ and $c(\{1,2\})=0.9$, which is less than $c(\{1\})+c(\{2\})=1.3$; thus $c$ is subadditive.

The reason is intuitively clear. Either of players 1 and 2 has a weight of one and puts it on his/her most preferable criterion, respectively, before forming a coalition. To increase their bargaining power, they choose to form a coalition, but after forming a coalition they are only given a total weight of one and put it on their most preferable criterion that maximizes their total evaluation. This is the primary reason for the subadditivity. The coalition of players 1 and 2 should be given weight of two since their total weights are two before forming a coalition. For example, in voting decisions, it is considered to be fair if the coalition is given two votes in total, in the case such that two voters each having one vote form a coalition.

On the basis of this consideration, we introduce a new approach to endogenously determine the weight vector. Each player is equally given a weight of one and he/she chooses a weight vector, the component of which is a portion of the weight given to each criterion. The sum of the weights given to all criteria must be one. Then by taking a simple average of all players' weight vectors, we obtain the weight vector that will be used to calculate each player's average contribution. Here "simple average" implies that each player has equal influence; in other words, players are treated equally. To illustrate the procedure, suppose, for example, in Table 1 , players 1 , 2 , and 3 choose weight vectors $(1 / 3,1 / 3,1 / 3),(1 / 2,1 / 2,0)$, and $(1 / 6,1 / 2,1 / 3)$, respectively. Then the weight vector for average contribution calculation is their simple average; that is, $((1 / 3+1 / 2+1 / 6) / 3,(1 / 3+1 / 2+1 / 2) / 3,(1 / 3+0+1 / 3) / 3)=$ $(1 / 3,4 / 9,2 / 9)$, and player 1's weighted average contribution is $6 / 10 \times 1 / 3+2 / 10 \times 4 / 9+1 / 10 \times 2 / 9=28 / 90$. Similarly the average contributions of players 2 and 3 are $36 / 90$ and 26/90, respectively.

By using this framework, we propose an alternative characteristic function form game. First we construct a strategic form game. Each player's strategy is a weight vector, and the payoff is his/her weighted average contribution calculated by using the simple average of all players' weight vectors. From this strategic form game, we derive a characteristic function form game following the procedure by von Neumann and Morgenstern [6], which will be explained in detail in the following sections.

\section{A Strategic Form DEA Game}

Let $N=\{1, \ldots, n\}$ be the set of players and let $M=\{1, \ldots, m\}$ be the set of criteria. The basic DEA model stated in Section 2 is as follows. Each player $j \in N$ chooses a weight vector $w^{j}=$ $\left(w_{1}^{j}, \ldots, w_{m}^{j}\right)$ with $w_{1}^{j}+\cdots+w_{m}^{j}=1, w_{i}^{j} \geq 0 \forall i \in M$, on the criteria so as to maximize the weighted sum of his/her relative evaluation indices, $\sum_{i=1}^{m} w_{i}^{j} c_{i j}$. The fixed amount of reward $E$ is shared by the players according to their weighted sums of the evaluation indices.

Therefore the strategic form game naturally reflecting the DEA model will be

$$
\left(N,\left\{W^{j}\right\}_{j \in N^{\prime}},\left\{f^{j}\right\}_{j \in N}\right)
$$

where $N=\{1, \ldots, n\}$ is the set of players, $W^{j}=\left\{w^{j}=\right.$ $\left.\left(w_{1}^{j}, \ldots, w_{m}^{j}\right) \mid w_{1}^{j}+\cdots+w_{m}^{j}=1, w_{i}^{j} \geq 0 \forall i \in M\right\}$ is the strategy set of player $j \in N$, and $f^{j}: W^{1} \times \cdots \times W^{n} \rightarrow \mathfrak{R}$ is the payoff function of player $j \in N$, which is given by

$$
f^{j}\left(w^{1}, \ldots, w^{n}\right)=\left(\sum_{i=1}^{m}\left(\frac{1}{n} \sum_{j=1}^{n} w_{i}^{j}\right) c_{i j}\right) E .
$$

Namely, the reward $E$ is shared by players in proportion to the weighted sum of their evaluation indices where the weights are the average weights over all players (another possible definition of $f^{j}$ is $f^{j}\left(w^{1}, \ldots, w^{n}\right)=\left(\sum_{i=1}^{m} w_{i}^{j} c_{i j} / \sum_{j^{\prime}=1}^{n}\left(\sum_{i=1}^{m} w_{i}^{j^{\prime}} c_{i j^{\prime}}\right)\right) E$; that is, the reward $E$ is shared by players in proportion to their weighted evaluation indices with their own weights. Both payoff functions lead to the same conclusions with respect to the solution concepts). Hereafter we call this game the strategic form DEA game. The equilibria in the strategic form game will be studied in Section 8 .

\section{TU DEA Game}

In the characteristic function form game theory, it is commonly considered that the characteristic function gives each coalition the value that the coalition members can surely win by themselves. Here the term "by themselves" means that they can win the value even if outsiders' behavior is least favorable to them. In our game, every player wishes to maximize his/her evaluation so as to maximize the share of $E$. Hence the least favorable behavior of outsiders is to minimize the coalition's evaluation, that is, the weighted sum of the players' evaluation indices inside the coalition. This behavior is also most favorable to the players outside the coalition since in our game players share a fixed amount of reward $E$. Namely, minimizing the coalition's evaluation leads to the maximization of the outsiders' evaluation. 
Therefore following von Neumann and Morgenstern [6], the characteristic function $v$ gives the following value $v(S)$ to each coalition $S \subseteq N$ :

$$
\begin{aligned}
v(S) & =\max _{\left(w^{j}\right)_{j \in S}} \min _{\left(w^{j}\right)_{j \in N \backslash S}} \sum_{j \in S} f^{j}\left(w^{1}, \ldots, w^{n}\right) \\
& =\left(\left(\frac{s}{n}\right) \times \max _{i=1, \ldots, m} \sum_{j \in S} c_{i j}+\left(\frac{n-s}{n}\right) \times \min _{i=1, \ldots, m} \sum_{j \in S} c_{i j}\right) E
\end{aligned}
$$

where $s$ is the number of players in $S$. From the perspective of the bargaining story we mentioned in the previous section, the worst situation for $S$ is when outsiders put their whole weights on the criterion with the lowest evaluation for the coalition. For example, in Table 2, coalition $\{1,2\}$ 's maximum and minimum evaluation indices are 0.9 and 0.2 . Therefore the value for coalition $\{1,2\}$ is properly measured by the weighted average of 0.9 and 0.2 with weights of $2 / 3$ and $1 / 3$, respectively. Thus we would propose $(2 / 3) \times 0.9+(1 / 3) \times 0.2=$ $2 / 3$ as the value of the characteristic function for coalition $\{1,2\}$. Similarly for the single player coalition $\{3\}$, the value would be $(2 / 3) \times 0.1+(1 / 3) \times 0.8=1 / 3$ since player 3 's maximum and minimum evaluation indices are 0.8 and 0.2 .

Note that $v(N)=E$ since $\sum_{j \in N} c_{i j}=1 \forall i=1, \ldots, m$. We call the characteristic function form game $(N, v)$ the TU DEA game since we allow for side payments among players assuming transferable utility. Nontransferable utility case will be studied in the future extension work.

\section{Properties of the TU DEA Game}

TU DEA games satisfy interesting properties. First TU DEA games are superadditive.

Definition 1 (superadditivity). A characteristic function form game $(N, v)$ is superadditive if

$$
v(S \cup T) \geq v(S)+v(T)
$$

holds for all $S, T \subseteq N$ with $S \cap T=\emptyset$.

Theorem 2. Let $(N, v)$ be a TU DEA game; then $(N, v)$ is superadditive.

Proof. It is obvious that

$$
\begin{aligned}
& \max _{i=1, \ldots, m} \sum_{j \in S \cup T} c_{i j} \geq \max _{i=1, \ldots, m} \sum_{j \in S} c_{i j}+\min _{i=1, \ldots, m} \sum_{j \in T} c_{i j}, \\
& \max _{i=1, \ldots, m} \sum_{j \in S \cup T} c_{i j} \geq \max _{i=1, \ldots, m} \sum_{j \in T} c_{i j}+\min _{i=1, \ldots, m} \sum_{j \in S} c_{i j} .
\end{aligned}
$$

Then by simple calculation, we can get

$$
\begin{aligned}
v(S \cup T) & -v(S)-v(T) \\
= & \left(\left(\frac{s+t}{n}\right) \times \max _{i=1, \ldots, m} \sum_{j \in S \cup T} c_{i j}+\left(\frac{n-s-t}{n}\right) \times \min _{i=1, \ldots, m} \sum_{j \in S \cup T} c_{i j}\right. \\
& -\left(\frac{s}{n}\right) \times \max _{i=1, \ldots, m} \sum_{j \in S} c_{i j}-\left(\frac{n-s}{n}\right) \times \min _{i=1, \ldots, m} \sum_{j \in S} c_{i j} \\
& \left.-\left(\frac{t}{n}\right) \times \max _{i=1, \ldots, m} \sum_{j \in T} c_{i j}-\left(\frac{n-t}{n}\right) \times \min _{i=1, \ldots, m} \sum_{j \in T} c_{i j}\right) E \\
\geq( & \left.\frac{n-s-t}{n}\right) \\
& \times\left(\min _{i=1, \ldots, m} \sum_{j \in S \cup T} c_{i j}-\min _{i=1, \ldots, m} \sum_{j \in S} c_{i j}-\min _{i=1, \ldots, m} \sum_{j \in T} c_{i j}\right) E
\end{aligned}
$$$$
\geq 0 \text {. }
$$

Furthermore TU DEA games are constant-sum.

Definition 3 (constant-sum). A characteristic function form game $(N, v)$ is constant-sum if

$$
v(S)+v(N \backslash S)=v(N)
$$

holds for all $S \subseteq N$.

Theorem 4. Let $(N, v)$ be a TU DEA game; then $(N, v)$ is constant-sum.

Proof. Since in our game $\sum_{j \in N} c_{i j}=1 \forall i=1, \ldots, m$, thus we have

$$
\begin{aligned}
& \max _{i=1, \ldots, m} \sum_{j \in S} c_{i j}+\min _{i=1, \ldots, m} \sum_{j \in N \backslash S} c_{i j}=1, \\
& \min _{i=1, \ldots, m} \sum_{j \in S} c_{i j}+\max _{i=1, \ldots, m} \sum_{j \in N \backslash S} c_{i j}=1 .
\end{aligned}
$$

Then it is quite simple to show that

$$
v(S)+v(N \backslash S)=\left(\frac{s}{n}+\frac{n-s}{n}\right) E=E=v(N) .
$$

\section{Solutions to the TU DEA Game}

7.1. Core. In characteristic function form games, solutions are considered within the concept of imputations.

Definition 5 (imputation). In a characteristic function form TU game $(N, v)$, a payoff vector $x=\left(x_{j}\right)_{j \in N}$ is called an imputation if it satisfies

(1) (group rationality) $\sum_{j \in N} x_{j}=v(N)$;

(2) (individual rationality) $x_{j} \geq v(\{j\}) \forall j \in N$.

The set of imputations of $(N, v)$ is denoted by $A(v)$. 
Definition 6 (core). The set

$$
C(v)=\left\{x \in A(v) \mid \sum_{j \in S} x_{j} \geq v(S) \forall S \subseteq N\right\}
$$

is called the core of $(N, v)$.

Definition 7 (inessentiality). A characteristic function form game $(N, v)$ is inessential if

$$
v(S)=\sum_{j \in S} v(\{j\})
$$

holds for all $S \subseteq N$. It is essential if $v(N)>\sum_{j \in N} v(\{j\})$.

If a characteristic function form game $(N, v)$ is inessential, then its imputation set is a singleton $(v(\{j\}))_{j \in N}$. Each player can receive the "safety" amount, guaranteeing the individual value being not worse off compared with the precoalition amount. Hence it is essential games that are of interest to us. The following theorem is well known; for the proof see Owen [8].

Theorem 8. Suppose that a characteristic function form game $(N, v)$ is constant-sum. Then if it is essential, its core $C(v)=\emptyset$.

As proved in the previous section, the TU DEA game $(N, v)$ is superadditive and constant-sum, and thus the core is nonempty only when the game is inessential, which is equal to the unique imputation set. The following theorem characterizes the inessential TU DEA game.

Theorem 9. A TU DEA game is inessential if and only if, for all $j \in N, c_{i j}=c_{i^{\prime} j}$ holds for all $i, i^{\prime}=1, \ldots, m$.

Proof. First let us see the sufficient condition. If $c_{i j}=c_{i^{\prime} j} \forall j \in$ $N \forall i, i^{\prime}=1, \ldots, m$, holds, by simple calculation

$$
\begin{aligned}
v(S) & =\left(\left(\frac{s}{n}\right) \times \max _{i=1, \ldots, m} \sum_{j \in S} c_{i j}+\left(\frac{n-s}{n}\right) \times \min _{i=1, \ldots, m} \sum_{j \in S} c_{i j}\right) E \\
& =\left(\sum_{j \in S} c_{i j}\right) E=\sum_{j \in S} v(\{j\}) .
\end{aligned}
$$

For the necessary condition, assume $\exists k \in N$ and $\exists \hat{i} \in M$ such that $c_{i k}>c_{i k}$ (or $c_{i k}<c_{i k}$; the proof is the same) and $c_{i k}=c_{i^{\prime} k} \forall i, i^{\prime} \in M \backslash\{\hat{i}\}$. For any other player $j \in$ $N \backslash\{k\}$, the condition in the theorem is satisfied, and thus, for the coalition $S^{\prime} \in\{S \subseteq N \mid\{k\} \quad \varsubsetneqq S\}$, we have $\max _{i=1, \ldots, m} \sum_{j \in S^{\prime}} c_{i j}>\min _{i=1, \ldots, m} \sum_{j \in S^{\prime}} c_{i j}$. Then

$$
\begin{aligned}
v\left(S^{\prime}\right) & =\left(\left(\frac{s^{\prime}}{n}\right) \times \max _{i=1, \ldots, m} \sum_{j \in S^{\prime}} c_{i j}+\left(\frac{n-s^{\prime}}{n}\right) \times \min _{i=1, \ldots, m} \sum_{j \in S^{\prime}} c_{i j}\right) E \\
& =\left(\sum_{j \in S^{\prime} \backslash\{k\}} c_{i j}+\left(\frac{s^{\prime}}{n}\right) c_{i k}+\left(\frac{n-s^{\prime}}{n}\right) c_{i k}\right) E \\
& >\left(\sum_{j \in S^{\prime} \backslash\{k\}} c_{i j}+\left(\frac{1}{n}\right) c_{i k}+\left(\frac{n-1}{n}\right) c_{i k}\right) E \\
& =\sum_{j \in S^{\prime}} v(\{j\}),
\end{aligned}
$$

which contradicts the definition of inessentiality.

Therefore in the TU DEA game, the core is nonempty if and only if the evaluation indices for all the criteria are identical for each player. It is usually not the case in reality and thus the core is generally empty. We can see that the allocation by the core concept is not of significant meaning for the TU DEA game.

7.2. Shapley Value. If the concept of the core is to give a set of stable imputations without distinguishing the most preferable payoff vector, although it might be empty, then the Shapley value is trying to assign the game a specific payoff vector. It is defined below.

Definition 10 (Shapley value). In a characteristic function form game $(N, v)$, for each $j \in N$,

$$
\phi_{j}(v)=\sum_{S \subseteq N, j \in S} \frac{(s-1) !(n-s) !}{n !}(v(S)-v(S \backslash\{j\}))
$$

is called the Shapley value for player $j$. The vector $\phi(v)=$ $\left(\phi_{j}(v)\right)_{j \in N}$ is called the Shapley value.

By applying the constant-sum property of the TU DEA game, the Shapley value can be represented in a much simpler form, which is also an exercise problem in Owen [8].

Theorem 11. In the TU DEA game $(N, v)$, the Shapley value is given by $\phi(v)=\left(\phi_{j}(v)\right)_{j \in N}$, where

$$
\phi_{j}(v)=2 \sum_{S \subseteq N, j \in S}\left(\frac{(s-1) !(n-s) !}{n !} v(S)\right)-v(N) \text {. }
$$

Proof. From the constant-sum property, we get $v(S \backslash\{j\})=$ $v(N)-v(N \backslash\{S \backslash\{j\}\})$, and we also know the combination equation can be represented as

$$
\left(\begin{array}{c}
n-1 \\
s-1
\end{array}\right)=\frac{(n-1) !}{(s-1) !(n-s) !} .
$$


Then the calculation is shown as follows:

$$
\begin{aligned}
\phi_{j}(v)= & \sum_{S \subseteq N, j \in S} \frac{(s-1) !(n-s) !}{n !} \\
& \times(v(S)-v(N)+v(N \backslash\{S \backslash\{j\}\})) \\
= & \sum_{S \subseteq N, j \in S} \frac{(s-1) !(n-s) !}{n !} \\
& \times(v(S)+v(N \backslash\{S \backslash\{j\}\})) \\
& -\sum_{s=1}^{n}\left(\begin{array}{l}
n-1 \\
s-1
\end{array}\right) \frac{(s-1) !(n-s) !}{n !} v(N) \\
= & 2 \sum_{S \subseteq N, j \in S}\left(\frac{(s-1) !(n-s) !}{n !} v(S)\right)-v(N) .
\end{aligned}
$$

For any $s=1, \ldots, n, S \cap\{N \backslash\{S \backslash\{j\}\}\}=\{j\}$ is always satisfied.

Example 12. Applying the simplified formula above and using the data set in Table 1 , the imputation by Shapley value is (9/30, 10/30,11/30), assuming $E=1$.

7.3. Nucleolus. Instead of applying a general axiomatization of fairness to a value function defined by the set of characteristic functions, the nucleolus looks at a fixed characteristic function, $v$, and tries to find an imputation $x=\left(x_{j}\right)_{j \in N}$ that minimizes the worst inequity. That is, we ask each coalition $S \subseteq N$ how dissatisfied it is with the proposed imputation $x$ and we try to minimize the maximum dissatisfaction.

Definition 13 (excess). As a measure of the inequity of an imputation $x=\left(x_{j}\right)_{j \in N}$ for a coalition $S \subseteq N$, the excess is defined as

$$
e(x, S)=v(S)-\sum_{j \in S} x_{j}
$$

which measures the amount by which coalition $S$ falls short of $v(S)$ by the proposed allocation $x$.

From the definition of the core, we immediately know that an imputation $x$ is in the core if and only if all of its excesses are negative or zero.

Define $O(x)$ as the vector of excesses arranged in nonincreasing order. On the vector $O(x)$ we use the lexicographic order, that is, $z>_{L} y$, if $\exists k \in\left\{1, \ldots, 2^{n}-2\right\}$, such that $z_{i}=$ $y_{i} \forall i \in\{1, \ldots, k-1\}$ and $z_{i}>y_{i} \forall i \in\left\{k, \ldots, 2^{n}-\right.$ $2\}$. We may omit the empty set and the grand coalition from consideration since their excesses are always zero. The nucleolus is an efficient allocation that minimizes $O(x)$ in the lexicographic ordering.

Definition 14 (nucleolus). Let $X=\left\{x=\left\{x_{j}\right\}_{j \in N} \mid \sum_{j=1}^{n} x_{j}=\right.$ $v(N)\}$ be the set of efficient allocations. One says that a vector $v \in X$ is a nucleolus if, for each $x \in X$, one has $O(v) \leq_{L} O(x)$.

The property such that $e(x, S)+e(x, N \backslash S)=0$ can reduce the computational complexity of the nucleolus, which can be proved by the constant-sum property of the TU DEA game. Thus we just have to check the excesses for half of the coalitions.

Example 15. Based on the data set in Table 1, consider an arbitrary point, say $(10 / 30,10 / 30,10 / 30) E$. Assuming $E=1$, the nucleolus $(9 / 30,10 / 30,11 / 30)$, which coincides with the imputation by Shapley value, can be calculated as in Table 3 .

Theorem 16. In the 3-player TU DEA game $(N, v)$, the allocations by the Shapley value and the nucleolus coincide.

Proof. Assuming $E=1$, the characteristic function for each coalition $S \in\{1,2,3\}$ is

$$
\begin{gathered}
v(\emptyset)=0, \\
v(\{1\})=\frac{1}{3} \max _{i \in M} c_{i 1}+\frac{2}{3} \min _{i \in M} c_{i 1}, \\
v(\{2\})=\frac{1}{3} \max _{i \in M} c_{i 2}+\frac{2}{3} \min _{i \in M} c_{i 2}, \\
v(\{3\})=\frac{1}{3} \max _{i \in M} c_{i 3}+\frac{2}{3} \min _{i \in M} c_{i 3}, \\
v(\{1,2\})=\frac{2}{3} \max _{i \in M}\left(c_{i 1}+c_{i 2}\right) \\
+\frac{1}{3} \min _{i \in M}\left(c_{i 1}+c_{i 2}\right)=1-v(\{3\}), \\
v(\{1,3\})=\frac{2}{3} \max _{i \in M}\left(c_{i 1}+c_{i 3}\right) \\
+\frac{1}{3} \min _{i \in M}\left(c_{i 1}+c_{i 3}\right)=1-v(\{2\}), \\
v(\{2,3\})=\frac{2}{3} \max _{i \in M}\left(c_{i 2}+c_{i 3}\right) \\
+\frac{1}{3} \min _{i \in M}\left(c_{i 2}+c_{i 3}\right)=1-v(\{1\}), \\
v(\{1,2,3\})=1 .
\end{gathered}
$$

The Shapley value allocation $\phi(v)$ is decided by

$$
\begin{aligned}
& \phi_{1}(v)=\frac{2}{3} v(\{1\})+\frac{1}{3}(v(\{1,2\})+v(\{1,3\}))-\frac{1}{3}, \\
& \phi_{2}(v)=\frac{2}{3} v(\{2\})+\frac{1}{3}(v(\{1,2\})+v(\{2,3\}))-\frac{1}{3}, \\
& \phi_{3}(v)=\frac{2}{3} v(\{3\})+\frac{1}{3}(v(\{1,3\})+v(\{2,3\}))-\frac{1}{3} .
\end{aligned}
$$


With the allocation above, the excess for each single player coalition is

$$
\begin{aligned}
& e(\phi(v),\{1\})=\frac{1}{3} v(\{1\})-\frac{1}{3}(v(\{1,2\})+v(\{1,3\}))+\frac{1}{3}, \\
& e(\phi(v),\{2\})=\frac{1}{3} v(\{2\})-\frac{1}{3}(v(\{1,2\})+v(\{2,3\}))+\frac{1}{3}, \\
& e(\phi(v),\{3\})=\frac{1}{3} v(\{3\})-\frac{1}{3}(v(\{1,3\})+v(\{2,3\}))+\frac{1}{3} .
\end{aligned}
$$

It can be easily verified that $e(\phi(v),\{1\})=e(\phi(v),\{2\})=$ $e(\phi(v),\{3\})$ is satisfied:

$$
\begin{aligned}
e(\phi(v),\{1\})-e(\phi(v),\{2\})= & \frac{1}{3}(v(\{1\})-v(\{2\})) \\
& -\frac{1}{3}(v(\{1,3\})-v(\{2,3\})) \\
= & 0, \\
e(\phi(v),\{1\})-e(\phi(v),\{3\})= & \frac{1}{3}(v(\{1\})-v(\{3\})) \\
& -\frac{1}{3}(v(\{1,2\})-v(\{2,3\})) \\
= & 0 .
\end{aligned}
$$

By the constant-sum property, any deviation from the allocation $\phi(v)$ will increase at least one of the excesses. Hence in the 3-player TU DEA game, the allocations by the Shapley value and the nucleolus always coincide.

For the cases that players are more than 3 , here is a counterexample showing that the theorem above does not necessarily hold.

Example 17. The Shapley value allocation is $(16 / 60,16 / 60$, $15 / 60,13 / 60$ ) with the data set in Table 4 . It can be easily verified that the maximum excess is not minimized with the allocation above, and hence the nucleolus allocation does not coincide with the Shapley value allocation in this example.

\section{Equilibria in the Strategic Form DEA Game}

In this section we study the equilibria in the strategic form DEA game, which is defined in Section 4.

\subsection{Nash Equilibrium}

Definition 18 (Nash equilibrium). In a strategic form game $\left(N,\left\{W^{j}\right\}_{j \in N},\left\{f^{j}\right\}_{j \in N}\right)$, a strategy combination $\left(w^{* 1}, \ldots, w^{* n}\right)$ is called a Nash equilibrium if for all $j \in N$

$$
f^{j}\left(w^{* j}, w^{*-j}\right) \geq f^{j}\left(w^{j}, w^{*-j}\right) \quad \forall w^{j} \in W^{j}
$$

holds. Here $w^{*-j}=\left(w^{* 1}, \ldots, w^{* j-1}, w^{* j+1}, \ldots, w^{* n}\right)$.
TABLE 3: The nucleolus calculation.

\begin{tabular}{lcccc}
\hline$S$ & $v(S)$ & $e(x, S)$ & $\left(\frac{10}{30}, \frac{10}{30}, \frac{10}{30}\right)$ & $\left(\frac{9}{30}, \frac{10}{30}, \frac{11}{30}\right)$ \\
\hline$\{1,2\}$ & $\frac{20}{30}$ & $\frac{20}{30}-x_{1}-x_{2}$ & 0 & $\frac{1}{30}$ \\
$\{1,3\}$ & $\frac{21}{30}$ & $\frac{21}{30}-x_{1}-x_{3}$ & $\frac{1}{30}$ & $\frac{1}{30}$ \\
$\{2,3\}$ & $\frac{22}{30}$ & $\frac{22}{30}-x_{2}-x_{3}$ & $\frac{2}{30}$ & $\frac{1}{30}$ \\
\hline
\end{tabular}

TABLE 4: Incoincidence of the Shapley value and nucleolus.

\begin{tabular}{lccccc}
\hline & Player 1 & Player 2 & Player 3 & Player 4 & Row-sum \\
\hline Criterion 1 & $6 / 10$ & $2 / 10$ & $1 / 10$ & $1 / 10$ & 1 \\
Criterion 2 & $2 / 10$ & $6 / 10$ & $1 / 10$ & $1 / 10$ & 1 \\
Criterion 3 & $1 / 10$ & $1 / 10$ & $5 / 10$ & $3 / 10$ & 1 \\
\hline
\end{tabular}

The Nash equilibrium is a strategy combination in which no player gains more by his/her unilateral deviation. Then we have the following theorem. That is, in Nash equilibrium, every player puts positive weights only on the criteria that give him/her the highest evaluation. Let $\overline{C^{j}}$ be the set of criteria that give player $j$ the maximum evaluation index; that is, $\overline{C^{j}}=\left\{i \in M \mid c_{i j} \geq c_{i^{\prime} j} \forall i^{\prime} \in M\right\}$.

Theorem 19. In a strategic form DEA game $\left(N,\left\{W^{j}\right\}_{j \in N}\right.$, $\left.\left\{f^{j}\right\}_{j \in N}\right)$, the Nash equilibrium is of the form

$$
w^{*}=\left(w^{* 1}, \ldots, w^{* n}\right), \quad w_{i}^{* j}=0 \quad \forall i \notin \overline{C^{j}} \forall j \in N .
$$

Proof. First let us prove the sufficient condition. By definition, we know that, for a strategy combination $\left(w^{* 1}, \ldots\right.$, $\left.w^{* n}\right)$ to be a Nash equilibrium, $f^{j}\left(w^{* j}, w^{*-j}\right) \geq f^{j}\left(w^{j}\right.$, $\left.w^{*-j}\right) \forall w^{j} \in W^{j}$ should be satisfied. Assume $w^{-j^{\prime}}=$ $\left(w^{1}, \ldots, w^{j^{\prime}-1}, w^{j^{\prime}+1}, \ldots, w^{n}\right)$ is determined; then for player $j^{\prime} \in N$

$$
f^{j^{\prime}}\left(w^{1}, \ldots, w^{n}\right)=\left(\sum_{i=1}^{m}\left(\frac{1}{n}\left(\sum_{j \in N, j \neq j^{\prime}} w_{i}^{j}+w_{i}^{j^{\prime}}\right)\right) c_{i j^{\prime}}\right) E .
$$

$c_{i j^{\prime}}(\forall i \in M)$ is given; in order to maximize $f^{j^{\prime}}\left(w^{1}, \ldots, w^{n}\right)$, player $j^{\prime}$ should choose a weight vector maximizing $\sum_{i=1}^{m}\left(w_{i}^{j^{\prime}} c_{i j^{\prime}}\right)$. It is obvious that only criteria with the highest evaluation indices should be assigned with positive weights. Applying this procedure for all other players, the Nash equilibrium should be $w^{*}=\left(w^{* 1}, \ldots\right.$, $\left.w^{* n}\right), w_{i}^{* j}=0 \forall j \in N \forall i \notin \overline{C^{j}}$.

For the necessary condition, assume that player $j^{\prime} \in N$ deviates from $w^{* j^{\prime}}$ to $w^{j^{\prime}}$ such that $\exists i^{\prime} \notin \overline{C^{j^{\prime}}}$ with $w_{i^{\prime}}^{j^{\prime}}>0$. Because $w_{1}^{j^{\prime}}+\cdots+w_{m}^{j^{\prime}}=1$, there must exist at least one criterion $i^{\prime \prime} \in \overline{C^{j^{\prime}}}$ to which the weight assigned becomes less 
than $w_{i^{\prime \prime}}^{* j^{\prime}}$; let us first assume such criteria set is a singleton, which means $w_{i^{\prime \prime}}^{j^{\prime}}=w_{i^{\prime \prime}}^{* j^{\prime}}-w_{i^{\prime}}^{j^{\prime}}$. Then

$$
\begin{aligned}
& f^{j^{\prime}}\left(w^{* 1}, \ldots, w^{* n}\right)-f^{j^{\prime}}\left(w^{* 1}, \ldots, w^{j^{\prime}}, \ldots, w^{* n}\right) \\
& \quad=\frac{1}{n} w_{i^{\prime}}^{j^{\prime}}\left(c_{i^{\prime \prime} j^{\prime}}-c_{i^{\prime} j^{\prime}}\right) E .
\end{aligned}
$$

By the definition of $\overline{C^{j^{\prime}}}$, we know that $c_{i^{\prime \prime} j^{\prime}}>c_{i^{\prime} j^{\prime}}$. Hence player $j^{\prime}$ gains less if he/she made this deviation. The proof for the condition when the weight assignments for multiple criteria become less than that before the deviation is similar.

Thus the Nash equilibrium exists, and the players choose putting positive weights only on the criteria with the highest evaluation indices.

8.2. Cooperative Behavior in the Strategic Form DEA Game. Next we examine players' cooperative behavior in the strategic form DEA game by using the concept of strong Nash equilibrium. First let us define the deviation.

Definition 20 (deviation). Take a strategy combination $w=$ $\left(w^{1}, \ldots, w^{n}\right)$. Coalition $T \subseteq N$ has a deviation $u^{T}=\left(u^{j}\right)_{j \in T}$ from $w$ if

$$
f^{j}\left(u^{T}, w^{-T}\right)>f^{j}(w) \quad \forall j \in T,
$$

where $w^{-T}=\left(w^{j}\right)_{j \in N \backslash T}$.

Definition 21 (strong Nash equilibrium). In a strategic form game $\left(N,\left\{W^{j}\right\}_{j \in N},\left\{f^{j}\right\}_{j \in N}\right)$, a strategy combination $w^{*}=$ $\left(w^{* 1}, \ldots, w^{* n}\right)$ is called a strong Nash equilibrium if no coalition $T \subseteq N$ has a deviation from $w^{*}$.

Thus in the strong Nash equilibrium, no group of players has an incentive to unilaterally deviate from the strategy combination. The strong Nash equilibrium must be a Nash equilibrium.

The strong Nash equilibrium does not always exist as shown in the following example.

Example 22. Suppose a strong Nash equilibrium exists with the data set in Table 5. Then it must be a Nash equilibrium; thus, by the theorem above, it must be of the form $w^{*}=$ $((0,1,0),(1,0,0),(1,0,0))$. Criterion 1 is chosen with probability $2 / 3$ and criterion 2 is chosen with probability $1 / 3$. By simple calculation, we get that players 1,2 , and 3 gain $5 / 30$, $6 / 30$, and 19/30, respectively. Suppose players 1 and 2 jointly deviate and choose $w^{\{1,2\}}=(0,0,2)$. Then since $w^{* 3}=$ $(1,0,0)$, criteria 1 and 3 are chosen with probabilities $1 / 3$ and $2 / 3$, respectively. Hence player 1 gains $6 / 30$ and player 2 gains $6.5 / 30$; both players are better off. Therefore there is no strong Nash equilibrium in this example.

We next analyze the coalition-proof Nash equilibrium. The strong Nash equilibrium assumes that the deviation is
TABLE 5: A counterexample for the existence of SNE.

\begin{tabular}{lcccc}
\hline & Player 1 & Player 2 & Player 3 & Row-sum \\
\hline Criterion 1 & $1 / 10$ & $2.5 / 10$ & $6.5 / 10$ & 1 \\
Criterion 2 & $3 / 10$ & $1 / 10$ & $6 / 10$ & 1 \\
Criterion 3 & $2.5 / 10$ & $2 / 10$ & $5.5 / 10$ & 1 \\
\hline
\end{tabular}

binding; that is, deviated coalition never breaks up. The coalition-proof Nash equilibrium supposes the possibility of further deviation inside deviated coalitions and considers only credible deviations, that is, deviations from which no further deviation takes place.

Definition 23 (credible deviation). We say $T \subseteq N$ has a credible deviation from a strategy profile $w=\left(w^{1}, \ldots, w^{n}\right)$ if (1) $T$ has a deviation $u^{T}=\left(u^{j}\right)_{j \in T} \in W^{T}$ from $w$ and (2) there is no $R \subsetneq T$ which has a credible deviation from $\left(u^{T}, w^{-T}\right)$. When $T=\{j\}, j$ has a credible deviation $u^{j}$ from $w$ if and only if $f^{j}\left(u^{j}, w^{-j}\right)>f^{j}(w)$. For $T$ with $|T|>1$, the definition of a credible deviation follows inductively.

Definition 24 (coalition-proof Nash equilibrium). In a strategic form game $\left(N,\left\{W^{j}\right\}_{j \in N},\left\{f^{j}\right\}_{j \in N}\right)$, a strategy combination $w^{*}=\left(w^{* 1}, \ldots, w^{* n}\right)$ is called a coalition-proof Nash equilibrium if no coalition $T \subseteq N$ has a credible deviation from $w^{*}$.

Same as the strong Nash equilibrium, the coalition-proof Nash equilibrium in the strategic form DEA game does not always exist as well. Here is a counterexample.

Example 25. Suppose that a coalition-proof Nash equilibrium exists with the data set in Table 6 , and $E=1$. Then it must be a Nash equilibrium; thus, by the theorem above, it must be of the form $w^{*}=\left(\left(w_{11}, 1-w_{11}, 0\right),\left(w_{12}, 0,1-\right.\right.$ $\left.\left.w_{12}\right),\left(0, w_{23}, 1-w_{23}\right)\right)$. Criterion 1 is chosen with probability $\left(w_{11}+w_{12}\right) / 3$, criterion 2 is chosen with probability $\left(1-w_{11}+\right.$ $\left.w_{23}\right) / 3$, and criterion 3 is chosen with probability $\left(2-w_{12}-\right.$ $\left.w_{23}\right) / 3$. By simple calculation following our basic proposal, we get that players 1,2 , and 3 gain $\left(2 w_{12}+2 w_{23}+8\right) / 30$, $\left(2 w_{11}-2 w_{23}+10\right) / 30$, and $\left(-2 w_{11}-2 w_{12}+12\right) / 30$, respectively.

Assume initially $w_{11} \in(0,1)$ and $w_{12} \in(0,1)$ as well. If players 1 and 2 form a coalition, they can jointly maximize their total as well as individual payoff by putting their whole weight on the first criterion, namely, letting $w_{11}=w_{12}=1$. Then players 1 and 2 can, respectively, gain $\left(2 w_{23}+10\right) / 30$ and $\left(-2 w_{23}+12\right) / 30$. Both players are better off. However, player 3 can only receive $8 / 30$.

We can also see that each player's payoff is irrelevant to its own choice on the weight vector; thus neither $\{1\}$ nor $\{2\}$ has a credible deviation from $w_{11}=w_{12}=1$. Hence the deviation above is credible.

Starting from this deviated strategy profile $((1,0,0)$, $\left.(1,0,0),\left(0, w_{23}, 1-w_{23}\right)\right)$, if players 1 and 3 form a coalition and choose to put their whole weight on the second criterion, then they can gain $12 / 30$ and 10/30, respectively. Both players 1 and 3 are better off, and this deviation is credible as well. 
TABLE 6: A counterexample for the existence of CPNE.

\begin{tabular}{lcccc}
\hline & Player 1 & Player 2 & Player 3 & Row-sum \\
\hline Criterion 1 & $4 / 10$ & $4 / 10$ & $2 / 10$ & 1 \\
Criterion 2 & $4 / 10$ & $2 / 10$ & $4 / 10$ & 1 \\
Criterion 3 & $2 / 10$ & $4 / 10$ & $4 / 10$ & 1 \\
\hline
\end{tabular}

In this manner, we can show that there is always a credible deviation from any Nash equilibrium. Hence the coalitionproof Nash equilibrium does not exist in this example.

Next we give a condition under which the coalition-proof Nash equilibrium exists and is uniquely determined.

Theorem 26. In a strategic form DEA game $\left(N,\left\{W^{j}\right\}_{j \in N}\right.$, $\left.\left\{f^{j}\right\}_{j \in N}\right)$, if $\overline{C^{j}}$ is a singleton set for each $j \in N$ and $w^{*}=$ $\left(w^{* 1}, \ldots, w^{* n}\right)$ is the Nash equilibrium, then $w^{*}$ is the unique coalition-proof Nash equilibrium, where $\overline{C^{j}}=\left\{i \in M \mid c_{i j} \geq\right.$ $\left.c_{i^{\prime} j} \forall i^{\prime} \in M\right\}$.

Proof. Let $c_{\overline{i(j)} j}$ denote the maximum evaluation index for player $j \in N$. If $\overline{C^{j}}$ is a singleton set, then $c_{\overline{i(j)} j}>$ $c_{i \prime j} \forall i^{\prime} \neq \overline{i(j)} \in M \forall j \in N$ must be satisfied. By Theorem 19, the unique Nash equilibrium is

$$
\begin{aligned}
w^{*} & =\left(w^{* 1}, \ldots, w^{* n}\right), \\
w_{\overline{i(j)}}^{* j} & =1, \quad w_{i^{\prime}}^{* j}=0 \quad \forall i^{\prime} \neq \overline{i(j)} \in M, \forall j \in N .
\end{aligned}
$$

Assume $\exists T \subseteq N$ such that $T$ has a credible deviation from $w^{*}$, and let $u^{T}=\left(u^{j}\right)_{j \in T} \in W^{T}$ denote the deviation. Then some $\{j\} \subsetneq T$ must have a deviation at $\left(u^{T}, w^{*-T}\right)$ by Theorem 19 . This deviation is a credible one by Definition 23. Therefore $u^{T}$ is not a credible deviation at $w^{*}$. Hence $w^{*}$ is the unique coalition-proof Nash equilibrium.

Theorem 26 shows that if for each player the criteria set giving him/her the maximum evaluation index is a singleton, then the coalition-proof Nash equilibrium exists and is uniquely determined.

\section{Concluding Remarks}

In this paper, we have improved the DEA min game proposed by Nakabayashi and Tone [1] by reassigning the total weight for the coalition members and developed a more natural, superadditive cooperative game scheme for this kind of problems. We first introduced a strategic form DEA game with the average weights over all players regarding each criterion in Section 4 and then defined the TU DEA game based on the definition of the strategic form game in Section 5 and studied its properties and solutions in Sections 6 and 7 , respectively. By applying the constant-sum property, we found the sufficient and necessary condition for the inessentiality of the TU DEA game, which is also the condition for a nonempty core. On showing that the core is usually empty in the TU DEA game, we next introduced the Shapley value and nucleolus and also proved that the allocations by these two concepts coincide with each other in the 3-player setting. Section 8 analyzed the equilibria for the strategic form DEA game. We gave a condition under which the coalition-proof Nash equilibrium exists and is uniquely determined.

Our future research subjects include the following:

(1) the solutions for the NTU DEA game, namely, the existence of $\alpha$-core and $\beta$-core;

(2) the role of other imputations, that is, the kernel, the proportional nucleolus, and so forth;

(3) the application of our proposed scheme in the voting game.

\section{Conflict of Interests}

The authors declare that there is no conflict of interests regarding the publication of this paper.

\section{References}

[1] K. Nakabayashi and K. Tone, "Egoist's dilemma: a DEA game," Omega, vol. 34, no. 2, pp. 135-148, 2006.

[2] L. Liang, W. D. Cook, and J. Zhu, "DEA models for two-stage processes: game approach and efficiency decomposition," Naval Research Logistics, vol. 55, no. 7, pp. 643-653, 2008.

[3] J. Wu, L. Liang, and Y. C. Zha, "Determination of the weights of ultimate cross efficiency based on the solution of nucleolus in cooperative game," System Engineering: Theory and Practice, vol. 28, no. 5, pp. 92-97, 2008.

[4] J. Wu, L. Liang, and F. Yang, "Determination of the weights for the ultimate cross efficiency using Shapley value in cooperative game," Expert Systems with Applications, vol. 36, no. 1, pp. 872876, 2009.

[5] J. Zhu, "A buyer-seller game model for selection and negotiation of purchasing bids: extensions and new models," European Journal of Operational Research, vol. 154, no. 1, pp. 150-156, 2004.

[6] J. von Neumann and O. Morgenstern, Theory of Games and Economic Behavior, Princeton University Press, Princeton, NJ, USA, 1944.

[7] A. Charnes, W. W. Cooper, and E. Rhodes, "Measuring the efficiency of decision making units," European Journal of Operational Research, vol. 2, no. 6, pp. 429-444, 1978.

[8] G. Owen, Game Theory, Emerald Group, Cambridge, Mass, USA, 3rd edition, 1995. 


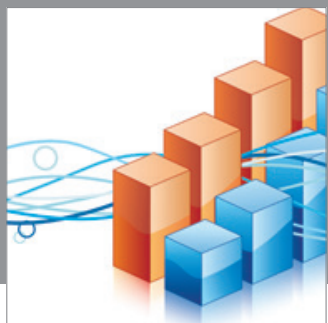

Advances in

Operations Research

mansans

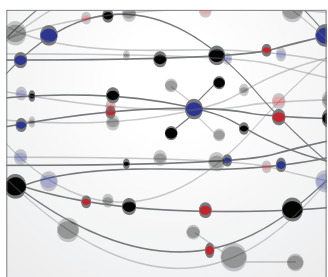

The Scientific World Journal
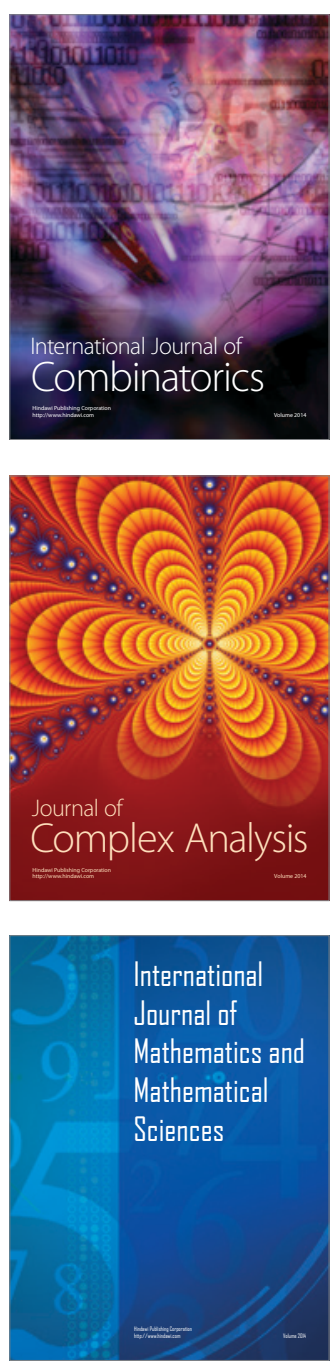
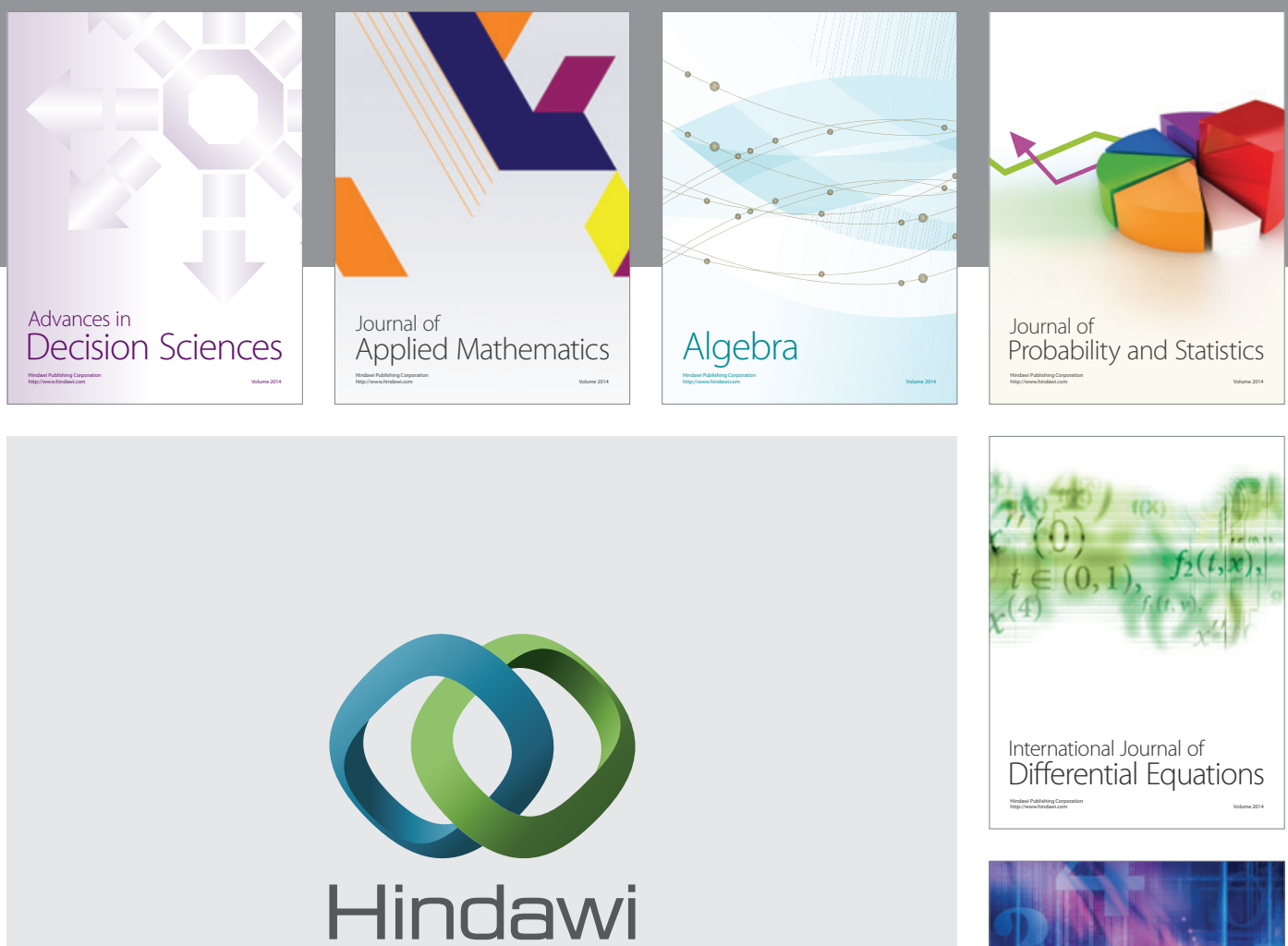

Submit your manuscripts at http://www.hindawi.com
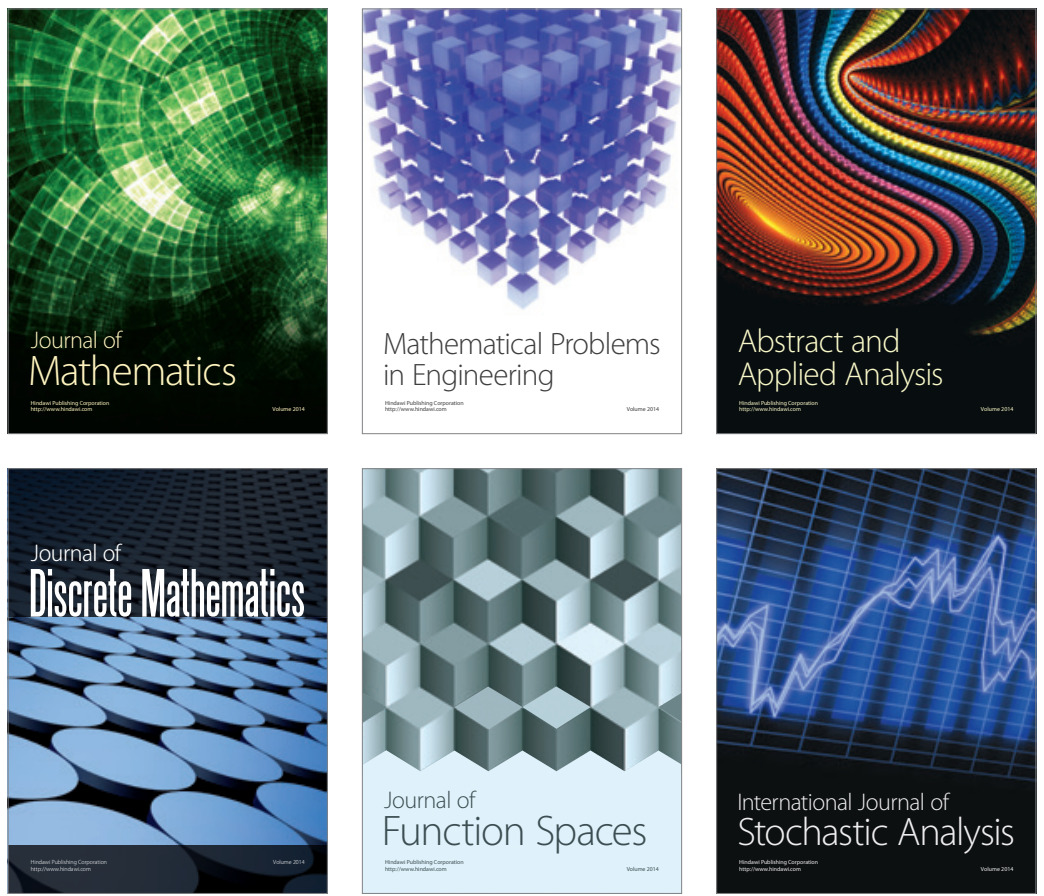

Journal of

Function Spaces

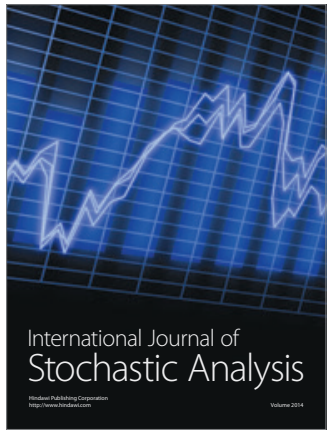

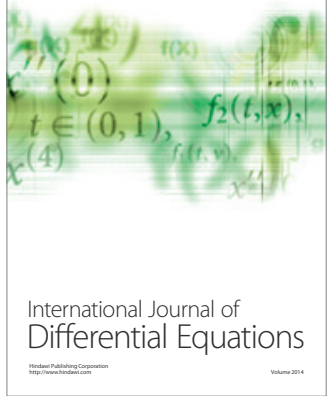
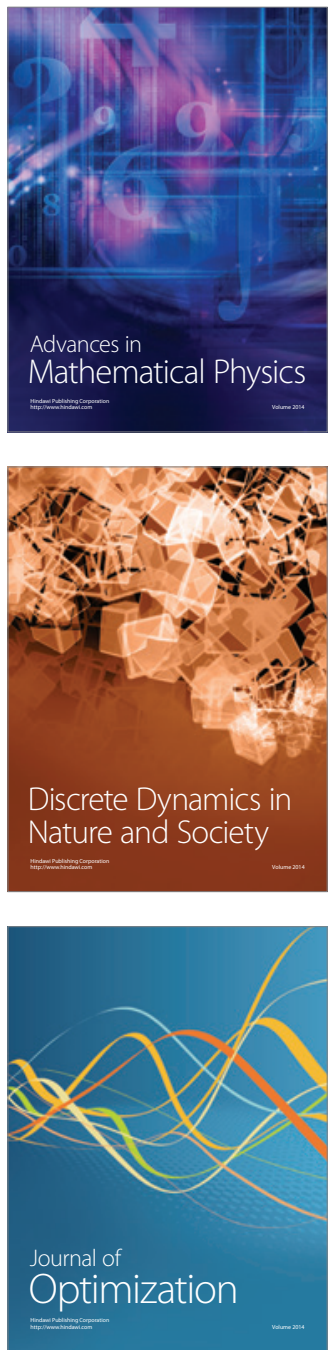